Rigo J, Ziegler EE (eds): Protein and Energy Requirements in Infancy and Childhood.

Nestlé Nutr Workshop Ser Pediatr Program, vol 58, pp 1-18,

Nestec Ltd., Vevey/S. Karger AG, Basel, (c) 2006.

\title{
Long-Term Consequences of Early Feeding on Later Obesity Risk
}

\author{
Berthold Koletzko
}

Division Metabolic Diseases and Nutritional Medicine, Dr. von Hauner Children's Hospital, Ludwig Maximilian University of Munich, Munich, Germany

\begin{abstract}
Some 30 years ago, Günter Dörner stated that the concentrations of hormones, metabolites and neurotransmitters during critical periods of early development will program disease risk in human adulthood, a concept that since has received enormous scientific support and broad attention. Evidence has also accumulated showing that early nutrition programs later obesity risk. Breastfeeding reduces the odds ratio for obesity at school age by about $20 \%$, relative to formula feeding, adjusted for biological and sociodemographic confounding variables. We propose that the protective effect of breastfeeding is explained at least in part by the induction of lower rates of infant weight gain, which may be related to differences in substrate intakes with breast milk and standard infant formulae. Protein intake per kilogram body weight is some 55-80\% higher in formula-fed than in breast-fed infants. We hypothesize that high early protein intakes in excess of metabolic requirements may enhance weight gain in infancy and later obesity risk (the 'early protein hypothesis'). The European Childhood Obesity Project is testing this hypothesis in a randomized double-blind intervention trial in more than 1,000 infants in 5 European countries. Infants that are not breast fed are randomized to formulae with higher or lower protein contents and are followed up to school age. If an effect of infant feeding habits on later obesity risk should be established, there is great potential for effective preventive intervention with a significant potential health benefit for the child and adult population.
\end{abstract}

Copyright @ 2006 Nestec Ltd., Vevey/S. Karger AG, Basel

Evidence is accumulating to show that metabolic events during critical time windows of pre- and postnatal development have marked modulating effects on health in later life, a concept often referred to as 'programming' or 'metabolic programming' [1]. It has been some three decades since Prof. Günter Dörner, 
then head of the Institute of Experimental Endocrinology at the Charité Hospital, Humboldt University at Berlin, Germany, first introduced the term 'programming' into the scientific literature to describe these phenomena [2]. In a visionary article reviewing a series of clinical and experimental data, Dörner [3] concluded that the concentrations of hormones, metabolites and neurotransmitters during critical early periods of development are capable of preprogramming brain development and, up to human adulthood, functional disturbances, diseases as well as syndromes of reproduction and metabolism. Dörner also proposed an interaction between the genetic material of the individual and environmental influences during early development to determine later function in adult life, a concept that has only recently been confirmed by experimental data [1, 4-6]. The concept has gained wide popularity following epidemiological studies documenting inverse relationships between body weight at birth and at age 1 year, respectively, and the risks of hypertension, diabetes and coronary heart disease in adulthood [7].

These observations have led to the hypothesis that maternal malnutrition during pregnancy would induce both fetal growth restriction and increased later disease risk, the fetal origins of the adult disease hypothesis [7]. However, this interpretation has recently been challenged based on the observation that low birth weight is associated with catch-up growth after birth, and accelerated weight gain by itself seems to be a risk factor for later disease [8]. Cole [9] substantiated the latter concept by multiple regression analysis of blood pressure outcomes on weights at different ages. Data from cohort studies in Brazil and the Philippines relating blood pressure in adolescence to weight through childhood showed small inverse weight effects in infancy, but early weight proved to be less important than weight and weight gain during adolescence [9].

Furthermore, Tu et al. [10] raised the possibility that evidence for the fetal origins of adult disease hypothesis might be a statistical artifact known as the 'reversal paradox', due in part to inappropriate statistical adjustment for variables on the causal pathway such as early weight gain and current body size. They performed computer simulations for three hypothetical relations between birth weight and adult blood pressure. The effect of statistically adjusting for different correlations between current weight and birth weight and between current weight and adult blood pressure was examined to assess their impact on associations between birth weight and blood pressure. When there was no genuine relation between birth weight and blood pressure, adjustment for current weight created an inverse association the size of which depended on the magnitude of the positive correlations between current weight and birth weight and between current weight and blood pressure. When there was a genuine inverse relation between birth weight and blood pressure, the association was exaggerated following adjustment for current weight, whereas a positive relation between birth weight and blood pressure could be reversed after adjusting for current weight. 
These questions need to be carefully elucidated, given the enormous preventive potential of optimizing early nutrition for long-term health, wellbeing and performance. Not only in view of the possible improvement in quality of life of individuals, but also in view of the enormous economic impact for societies, major investments in research are justified to explore the mechanisms and effects of early nutritional programming on long-term health. Today a large number of scientific investigators study these issues. In Europe many leading research groups collaborate in the international research cluster EARNEST (www.metabolic-programming.org) supported by the European Union.

\section{Programming of Later Obesity Risk}

Childhood obesity is now considered a global epidemic in view of the alarming increase in its prevalence and severity, not only in affluent but also in less privileged childhood populations worldwide [11-13]. Serious shortand long-term consequences of childhood obesity arise in terms of damage to quality of life, performance, health and life expectancy. In addition, the size of the obesity epidemic is estimated to create huge costs for society due to loss of productivity and ensuing costs for health care and social security. Faced with the size of the problem, widely available and effective medical management of children who are already obese is needed, but at present the results of available treatment concepts are far less than satisfactory, and costs are high [14]. A recent Cochrane review on interventions for treating obesity in children found that no conclusions on the effects of treatment strategies and their components can be drawn with confidence [15]. Thus, in the present situation the emphasis must be put on the development, evaluation and implementation of effective primary prevention of obesity. Several indications exist that modification of infant nutrition may offer opportunities for contributing to the prevention of later obesity risk [1].

\section{Early Growth and Later Obesity Risk}

Already in the 1950s, McCance and Widdowson showed that alterations in early growth by manipulating the feeding conditions of piglets during sensitive pre- and postnatal periods predetermined their ultimate weight in adulthood [16]. In humans high birth weight has been proposed as a risk factor for later overweight $[17,18]$, which could reflect both the roles of genetics and early priming by intrauterine environment. Additionally recent studies pointed to further priming of childhood overweight in the first 2 years of life by a high postnatal weight gain [19-22]. In order to assess the best anthropometric predictor from birth to 2 years for later overweight, we performed a cohort study in Bavaria, southern Germany, on 4,235 German children aged 5-6 years 
overall predictor of overweight at school entry compared to other anthropometric markers and time intervals.

In contrast to our results, Stettler et al. [25] recently proposed that the weight change in the first week after birth might be critical for modulating later obesity risk. They analyzed data obtained in interviews with 653 adults aged 20-32 years who had participated as infants in controlled but not randomized trials testing the feeding of infant formulae based on cows milk or soy proteins from 8 to 112 days of life. For an additional $100 \mathrm{~g}$ weight gain between birth and age 8 days, the odds ratio for being overweight in young adulthood (BMI $>25$ ) increased significantly by $20 \%$, whereas there was only a trend to an increase by $6 \%$ for $100 \mathrm{~g}$ weight gain between birth and age 122 days. However, the data on which the authors' conclusions are based appear questionable because the dimension of weight gain between birth and either 8 or 122 days, respectively, is markedly different. Thus the use of one and the same absolute weight gain (100 g) as the denominator appears inappropriate. In fact, the recalculation of their data to percentage weight change indicates that a 10\% change in weight gain between birth and day 8 (approx. 20.5 g) increases adult overweight risk by only $4.1 \%$, whereas a $10 \%$ higher weight gain between birth and day 112 (approx. 309 g) leads to a 18.5\% higher risk of adult overweight. Thus, also these data seem to support that it is growth rates over a long period in infancy that are predictive of later obesity.

The rate of weight gain in the first 2 years of life is influenced by the genetic factors of the individual and its mother, birth weight, metabolic influences during pregnancy, health and disease factors, such as the occurrence of infections, and not least by diet and nutrient supply.

\section{Protective Effects of Breastfeeding against Later Obesity}

It has long been known that populations of infants fed breast milk or formula differ in their growth kinetics, with formula-fed infants showing higher weight and length gains [26]. Based on a systematic review of 19 studies in affluent populations, Dewey [27] concluded that by the age of 12 months, the cumulative difference in body weight amounts to approximately $400 \mathrm{~g}$ in infants breast fed for 9 months and as much as 600-650 g in infants who are breast fed for 12 months. Given this very large effect of the mode of feeding on early weight gain, we attempted to study whether breastfeeding might also confer protection against later obesity risk.

In a cross-sectional survey in Bavaria, Germany, we assessed the impact of breastfeeding on the risk of obesity and the risk of being overweight in children at the time of school entry [28]. Routine data were collected on the height and weight of 134,577 children participating in the obligatory health examination at the time of school entry in Bavaria. In a sub-sample of 13,345 children, early feeding, diet, and lifestyle factors were assessed using responses to a 


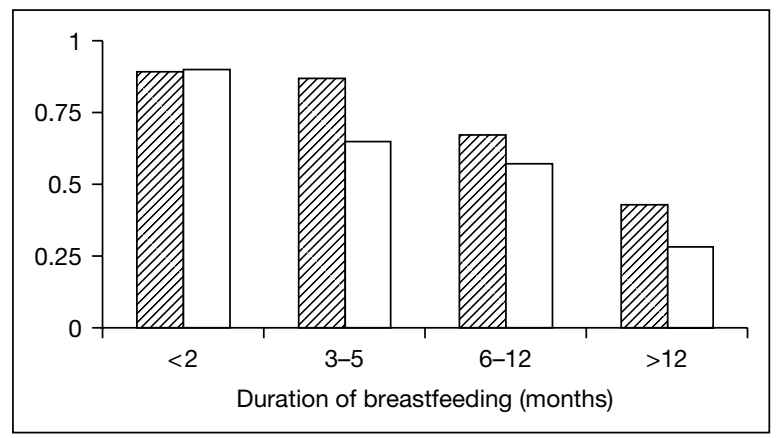

Fig. 1. The adjusted odds ratio for overweight ( $\square)$ and obesity ( $\square$ ) at school entry decreases with increasing duration of breastfeeding. Adapted from von Kries et al. [28].

questionnaire completed by the parents. The data of 9,357 children aged 5 and 6 years who had German nationality were included in the final analysis. Being overweight was defined as having a BMI above the 90th percentile and obesity was defined as a BMI above the 97th percentile of all 134,577 German children examined in this year. The prevalence of obesity in children who had never been breast fed was $4.5 \%$ as compared with $2.8 \%$ in breast-fed children. A clear dose-response effect was identified for the duration of breastfeeding on the prevalence of obesity: the prevalence was 3.8\% for 2 months of exclusive breastfeeding, $2.3 \%$ for $3-5$ months, $1.7 \%$ for $6-12$ months, and $0.8 \%$ for more than 12 months. Similar relations were found with the prevalence of being overweight. The protective effect of breastfeeding was not attributable to differences in social class or lifestyle. After adjusting for potential confounding factors, breastfeeding remained a significant protective factor against the development of obesity (odds ratio $0.75,95 \%$ CI $0.57-0.98$ ) and being overweight (odds ratio $0.79,95 \%$ CI 0.68-0.93), again with a clear dose-response relationship between the duration of breastfeeding and later risk of overweight and obesity, respectively (fig. 1). We conclude that promoting prolonged breastfeeding may help decrease the prevalence of obesity.

Following our publication, a number of other investigators studied this relationship in data from various cohorts around the world. We thus performed a systematic review and meta-analysis of published epidemiological studies (cohort, case-control or cross-sectional studies) comparing early feeding mode and adjusting for potential confounding factors [29]. Electronic databases were searched and reference lists of relevant articles were checked. Calculations of pooled estimates were conducted in fixed-effects and random-effects models. Heterogeneity was tested by Q-test. Publication bias was assessed from funnel plots and by a linear regression method. Nine studies with more than 69,000 participants met the inclusion criteria. The metaanalysis showed that breastfeeding reduced the risk of obesity in childhood 


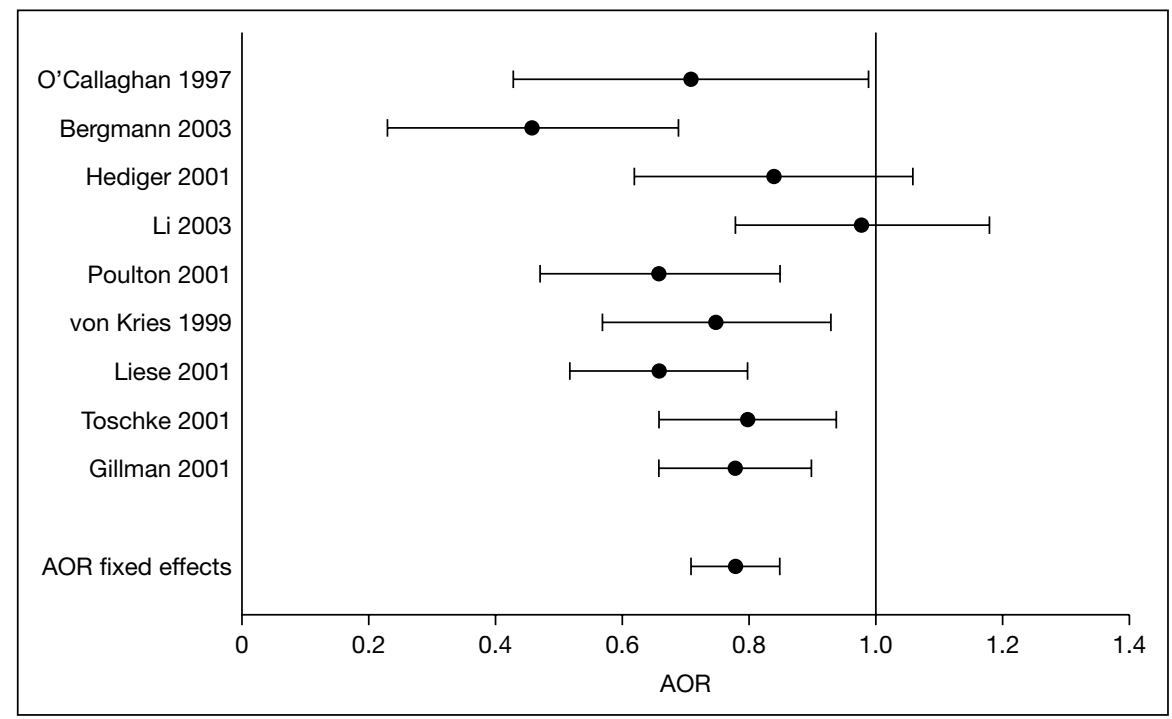

Fig. 2. Effect of breastfeeding vs. formula feeding on childhood obesity: covariateadjusted odds ratios of 9 studies and pooled adjusted odds ratio (AOR). Adapted from Owen et al. [30].

significantly. The adjusted odds ratio was 0.78 (95\% CI 0.71-0.85) in the fixed model (fig. 2). The assumption of homogeneity of the results of the studies included could not be refuted (Q-test for heterogeneity, $p>0.3$ ), stratified analyses showed no differences regarding different study types, age groups, definition of breastfeeding or obesity and number of confounding factors adjusted for. A dose-dependent effect of breastfeeding duration on the prevalence of obesity was reported in 4 studies. Funnel plot regression gave no indication of publication bias. Another recently published meta-analysis confirmed a protective effect of breastfeeding, but reported a smaller effect size with an odds ratio of 0.87 , primarily influenced by the results of one publication from the USA with a very large sample size [30].

\section{Potential Causes for the Protective Effects of Breastfeeding on Later Obesity}

A number of hypotheses can be raised on the potential causes of a protective effect of breastfeeding. Even though the inverse relationship of both breastfeeding and breastfeeding duration with later obesity persists after adjustment for measurable confounding variables, residual confounding cannot be fully excluded. Since one cannot randomize healthy babies to either breast milk or formula feeding for ethical and practical reasons, undisputable 
proof for a protective effect of breastfeeding can hardly be obtained. However, the consistent results of many studies and the dose-response effect between the duration of breastfeeding and the later reduction of obesity risk observed in a number of studies make an effect of breastfeeding highly likely.

Differences in feeding behavior and mother-child interactions between populations of breast- and formula-fed infants might play a role. Breast-fed infants show a different suckling pattern and a higher suckling frequency [31, 32]. Breast-fed infants seem to have a greater degree of control on meal sizes and intervals than those fed formula. Sievers et al. [33] monitored marked differences in feeding patterns, with a $20-30 \%$ higher feeding volume of formulafed infants after 6 weeks of life as well as a smaller number of total meals and of nightly meals in bottle-fed babies at 4 months of age. Such differences may modulate later body size. Agras et al. [34] reported that early feeding patterns were predictive of BMI at 3 years of age, with high-pressure sucking measured in the laboratory at 2 and 4 weeks of age (denoting a vigorous feeding style) associated with a greater degree of adiposity in toddlers.

In contrast to infant formula, breast milk shows marked variation in its taste and smell from day to day, and even from meal to meal, depending on maternal dietary habits and other metabolic factors. Since early taste experience in infancy has been shown to favor later consumption of foods with the same taste [35], it is conceivable that breast-fed infants might be programmed to different food selection and dietary habits in later life.

Moreover, breastfeeding appears to enhance the emotional bonding of the mother to her child, mediated in part by the stimulation of maternal oxytocin release by infant suckling, and breastfeeding has been shown to lead to decreased neuroendocrine response to stressors and decreased negative mood in the mothers $[36,37]$. These effects of breastfeeding might well have repercussions on the interaction between mother and child and healthrelated behaviors. These and further behavioral hypotheses are plausible and attractive, but are difficult to test experimentally, thus for the time being they remain somewhat speculative.

The mode of infant feeding at the breast cannot be copied by human milk substitutes, but if the protective effects of breastfeeding were related to the compositional aspects of breast milk and to the nature of substrate supply, such benefits might potentially be extended, at least in part, also to formulafed populations by appropriate modifications to infant formula composition. Promising hypotheses can be deducted from studies evaluating physiological differences of breast- and bottle-fed infants. The higher growth rates observed in populations of formula-fed infants compared to infants fed breast milk are most likely due to differences in metabolizable substrate intakes.

Infant formulae have a higher average caloric density (kcal/100 ml) than the mean values for breast milk, and energy supplies per kilogram body weight to formula-fed infants are 10-18\% higher than those to breast-fed babies between 3 and 12 months of age [38]. Even larger is the difference in 


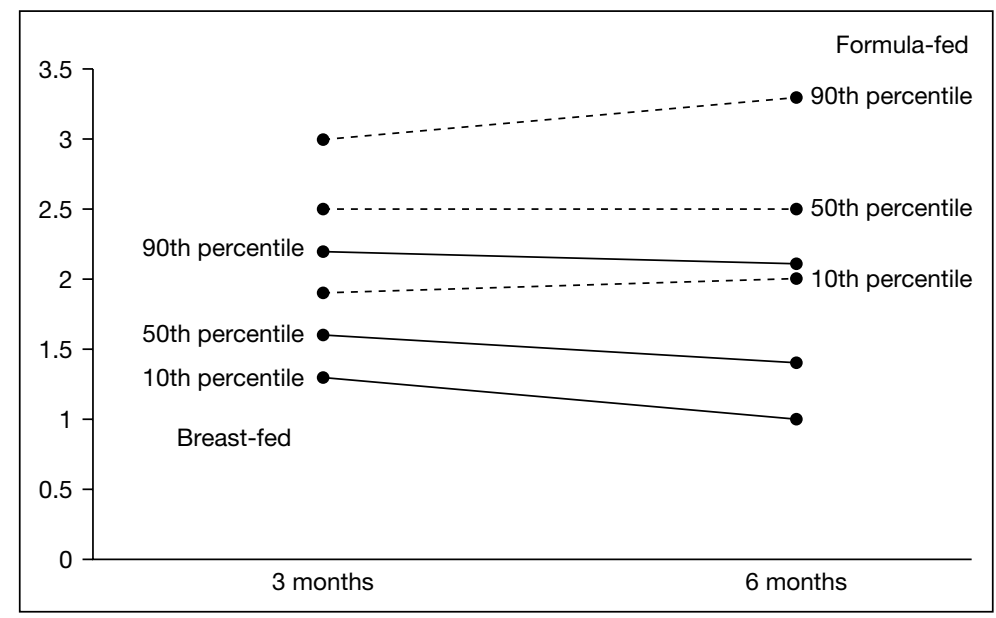

Fig. 3. Protein intake at ages 3 and 6 months (g/kg body weight, median and 90th/10th percentiles) in breast-fed and formula-fed infants, respectively, participating in the German DONALD study. Drawn from data from Alexy et al. [39].

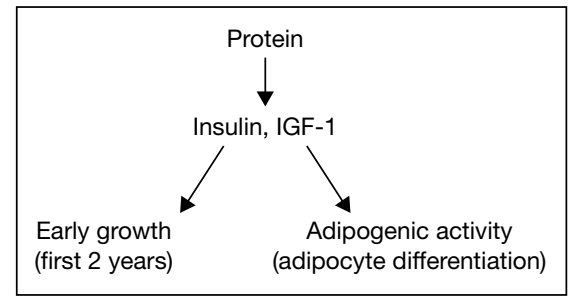

Fig. 4. Infant protein intake in excess of metabolic requirements may stimulate the secretion of insulin and insulin-like growth factor-1 (IGF-1) which, in turn, can enhance both growth during the first 2 years of life as well as adipogenic activity and adipocyte differentiation.

protein intake per kilogram body weight, which is $55-80 \%$ higher in formulathan in breast-fed infants (fig. 3) [39].

In rats, prenatal exposure to high protein decreased energy expenditure and increased later adiposity [40], and a high postnatal protein and nutrient supply led to higher adult body fat deposition [41] and increased adult weight by $10-40 \%$ [42]. A high protein intake in excess of metabolic requirements may enhance the secretion of insulin and insulin-like growth factor-1 (IGF-1). Indeed, infants fed formula had far greater postprandial levels of insulin on day 6 of life than infants fed cow's milk-based formula [43]. High insulin and IGF-1 values can enhance both growth during the first 2 years of life $[44,45]$ as well as adipogenic activity and adipocyte differentiation [46] (fig. 4). High protein intakes may also decrease human growth hormone secretion and lipolysis.

Indeed, high protein intakes in early childhood, but not the intakes of energy, fat or carbohydrate, were significantly related to an early occurrence 
of adiposity rebound and to high childhood BMI, corrected for parental BMI [47-50]. Thus, we hypothesize that a high protein intake with infant formula, in excess of metabolic requirements, may predispose to an increased obesity risk in later life (early protein hypothesis).

\section{Testing the Early Protein Hypothesis: The European Childhood Obesity Project}

In addition to prospective epidemiological and experimental studies, human intervention trials are needed to test this 'early protein hypothesis'. Therefore, we have set up the European Childhood Obesity Project (www. childhood-obesity.org) funded by the European Commission's 5th Framework Research Programme to test, in a randomized double-blind intervention trial, whether variation in protein intakes during the first year of life affects growth kinetics and later obesity risk. This trial is being conducted in 5 European countries which differ substantially in their prevalence of adult obesity and also in the nutritional characteristics of the habitual diet of infants and children, in particular in protein supply with complementary feeding, i.e. Germany (project and center coordinator Prof. Berthold Koletzko, Munich), Belgium (center coordinator Prof. Philippe Goyens, Brussels), Italy (center coordinator Prof. Marcello Giovannini, Milan), Poland (center coordinator Prof. Jerzy Socha, Warsaw) and Spain (center coordinator Dr. Ricardo Closa, Tarragona). Therefore the trial offers the opportunity to combine a multicenter intervention trial on infant formulae which differ in their balance of protein and fat (Bledina, Steenvoorde, France), with an epidemiological observation study which can assess the balance of protein and fat in the overall early diet. This approach will enable us to assess the effect of variables which differ substantially within Europe, as well as allowing the intervention trial results to be analyzed within centers. The inclusion of a group of breast-fed infants in each center will also allow an epidemiological comparison of the effects of breastfeeding and formula feeding in the different countries. This approach will provide the opportunity for an external validation of the underlying hypothesis.

Growth from birth to age 2 years, a marker of later obesity risk, was chosen as the primary outcome variable. In addition, a variety of further variables are measured, including detailed data on diet, lifestyle and behavior, biochemical and endocrine markers, markers of renal function, and others (fig. 5). Randomization and data collection are performed via the internet based on uniform electronic case report forms, using specially developed information technology architecture with a central database and 12 remote data entry stations as well as dedicated software that enables secure data protection. Mechanisms for quality assurance have also been established. Data input and transfer to the central database are supervised by a contract research organization participating in the project. 


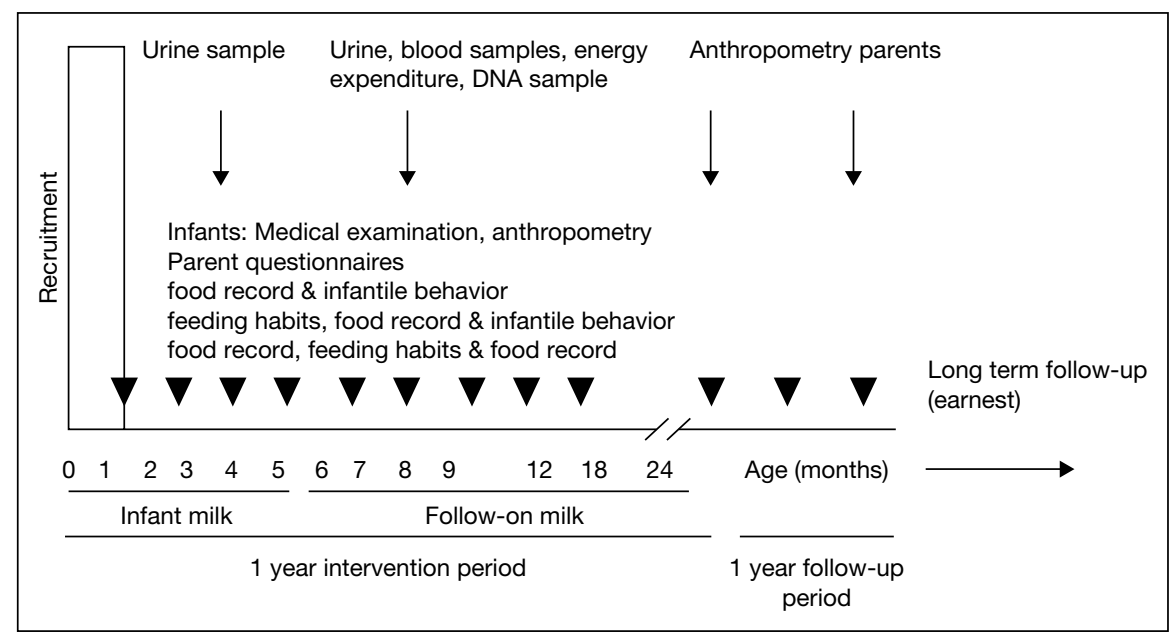

Fig. 5. Summary of the study design of the European Childhood Obesity Project. Infants are randomized to infant and follow-on formulae with higher or lower protein intakes, respectively, from the neonatal age for the first year of life. Diet, behavior, growth, metabolic and endocrine markers, and a number of other variables are monitored during regular follow-up visits. Adapted from Koletzko et al. [1].

The intervention trial started on October 1, 2002, and recruitment was completed on June 30, 2004. Following the study protocol and the requirements to report the first results to the $\mathrm{EU}$ at the end of the first funding periods, the study will not be blinded in the second half of 2006 to allow first data evaluations. However, the children participating and their families will be invited for further follow-up until 2010 as part of the EU 6th Framework Research Project, EARNEST, which investigates long-term health effects or early nutrition (www.metabolic-programming.org). Therefore, the European Childhood Obesity Project offers unique and exciting opportunities for evaluating the effects of early diet on long-term health in later life. If an effect of infant feeding habits and, in particular, of high protein intakes on long-term growth, development of later body composition and obesity risk can be established, there is great potential for effective preventive intervention by modification of the composition and use of dietary products for infants. Thus, the expected results might have a very direct application with a significant potential health benefit for the child and adult population.

\section{Acknowledgments}

The authors thank the participating families and all project partners for their enthusiastic support of the project work. The studies reported herein have been 
carried out with partial financial support from the Commission of the European Communities, specifically the RTD program 'Quality of Life and Management of Living Resources', within the 5th Framework Programme, research grants No. QLRT-200100389 and QLK1-CT-2002-30582, and the 6th Framework Programme, contract No. 007036. This manuscript does not necessarily reflect the views of the Commission and in no way anticipates the future policy in this area. Additional support from the International Danone Institutes, the University of Munich, and the Child Health Foundation is gratefully acknowledged.

\section{Statement on Conflict of Interest}

The author declares that there is no conflict of interest according to the definitions of the International Committee of Medical Journal Editors (http://www. icmje.org/).

\section{References}

1 Koletzko B, Akerblom H, Dodds PF, Ashwell M: Early nutrition and its later consequences: new opportunities. Adv Exp Med Biol 2005;569:1-237.

2 Koletzko B: Developmental origins of adult disease: Barker's or Dörner's hypothesis? Am J Hum Biol 2005;17:381-382.

3 Dörner G: Perinatal hormone levels and brain organization; in Stumpf WE, Grant LD (eds): Anatomical Neuroendocrinology. Basel, Karger, 1975, pp 245-252.

4 Schmidt I, Schoelch C, Ziska T, et al: Interaction of genetic and environmental programming of the leptin system and of obesity disposition. Physiol Genomics 2000;3:113-120.

5 Ozanne SE, Fernandez-Twinn D, Hales CN: Fetal growth and adult diseases. Semin Perinatol 2004;28:81-87.

6 Plagemann A: 'Fetal programming' and 'functional teratogenesis': on epigenetic mechanisms and prevention of perinatally acquired lasting health risks. J Perinat Med 2004;32:297-305.

7 Barker DJ, Osmond C, Golding J, et al: Growth in utero, blood pressure in childhood and adult life, and mortality from cardiovascular disease. BMJ 1989;298:564-567.

8 Singhal A, Lucas A: Early origins of cardiovascular disease: is there a unifying hypothesis? Lancet 2004;363:1642-1645.

9 Cole TJ: Modeling postnatal exposures and their interactions with birth size. J Nutr 2004;134:201-204.

10 Tu YK, West R, Ellison GT, Gilthorpe MS: Why evidence for the fetal origins of adult disease might be a statistical artifact: the 'reversal paradox' for the relation between birth weight and blood pressure in later life. Am J Epidemiol 2005;161:27-32.

11 Koletzko B, Girardet JP, Klish W, Tabacco O: Obesity in children and adolescents worldwide: current views and future directions. J Pediatr Gastroenterol Nutr 2002;35:S205-S212.

12 Fisberg M, Baur L, Chen W, et al: Childhood obesity - a global perspective. J Pediatr Gastroenterol Nutr 2004;39:S678-S687.

13 Koletzko B, de la Guéronnière V, Toschke AM, von Kries R: Nutrition in children and adolescents in Europe: what is the scientific basis? Introduction. Br J Nutr 2004;92(suppl 2): S67-S73.

14 Koletzko B: Childhood obesity: time for treatment or prevention? Eur J Lipid Sci Technol 2004;106:287-288.

15 Summerbell CD, Ashton V, Campbell KJ, et al: Interventions for treating obesity in children. Cochrane review. Cochrane Library, 2004, Issue 1.

16 Ashwell M (ed): McCance and Widdowson. A Scientific Partnership for 60 Years. London, British Nutrition Foundation, 1993.

17 Eriksson J, Forsen T, Osmond C, Barker D: Obesity from cradle to grave. Int J Obes Relat Metab Disord 2003;27:722-727.

18 Binkin NJ, Yip R, Fleshood L, Trowbridge FL: Birth weight and childhood growth. Pediatrics 1988;82:828-834. 
19 Ong KK, Ahmed ML, Emmett PM, et al: Association between postnatal catch-up growth and obesity in childhood: prospective cohort study. BMJ 2000;320:967-971.

20 Stettler N, Zemel BS, Kumanyika S, Stallings VA: Infant weight gain and childhood overweight status in a multicenter, cohort study. Pediatrics 2002;109:194-199.

21 Stettler N, Kumanyika SK, Katz SH, et al: Rapid weight gain during infancy and obesity in young adulthood in a cohort of African Americans. Am J Clin Nutr 2003;77:1374-1378.

22 Stettler N, Stallings VA, Troxel AB, et al: Weight gain in the first week of life and overweight in adulthood: a cohort study of European American subjects fed infant formula. Circulation 2005;111:1897-1903.

23 Toschke AM, Grote V, Koletzko B, von Kries R: Identifying children at high risk for overweight at school entry by weight gain during the first 2 years. Arch Pediatr Adolesc Med 2004;158:449-452.

24 Toschke AM, Vignerova J, Lhotska L, et al: Overweight and obesity in 6- to 14-year-old Czech children in 1991: protective effect of breastfeeding. J Pediatr 2002;141:764-769.

25 Stalling VA, Troxel AB, Zhao J, et al: Weight gain in the first week of life and overweight in adulthood: a cohort study of European American subjects fed infant formula. Circulation 2005;111: 1897-1903.

26 Kramer MS, Guo T, Platt RW, et al, Promotion of Breastfeeding Intervention Trials Study Group: Feeding effects on growth during infancy. J Pediatr 2004;145:600-605.

27 Dewey KG: Growth characteristics of breast-fed compared to formula-fed infants. Biol Neonate 1998;74:94-105.

28 von Kries R, Koletzko B, Sauerwald T, et al: Breastfeeding and obesity: cross sectional study. BMJ 1999;319:147-150.

29 Arenz S, Rückerl R, Koletzko B, von Kries R: Breast-feeding and childhood obesity. A systematic review. Int J Obesity 2004;28:1247-1256.

30 Owen CG, Martin RM, Whincup PH, et al: Effect of infant feeding on the risk of obesity across the life course: a quantitative review of published evidence. Pediatrics 2005;115:1367-1377.

31 Bosma JF, Hepburn LG, Josell SD, Baker K: Ultrasound demonstration of tongue motions during suckle feeding. Dev Med Child Neurol 1990;32:223-229.

32 Mathew OP, Bhatia J: Sucking and breathing patterns during breast- and bottle-feeding in term neonates. Effects of nutrient delivery and composition. Am J Dis Child 1989;143:588-592.

33 Sievers E, Oldigs HD, Santer R, Schaub J: Feeding patterns in breast-fed and formula-fed infants. Ann Nutr Metab 2002;46:243-248.

34 Agras WS, Kraemer HC, Berkowitz RI, Hammer LD: Influence of early feeding style on adiposity at 6 years of age. J Pediatr 1990;116:805-809.

35 Mennella JA, Jagnow CP, Beauchamp GK: Prenatal and postnatal flavor learning by human infants. Pediatrics 2001;107:E88.

36 Klaus M: Mother and infant: early emotional ties. Pediatrics 1998;102(suppl E):1244-1246.

37 Mezzacappa ES: Breastfeeding and maternal stress response and health. Nutr Rev 2004;62: 261-268.

38 Heinig MJ, Nommsen LA, Peerson JM, et al: Energy and protein intakes of breast-fed and formula-fed infants during the first year of life and their association with growth velocity: the DARLING Study. Am J Clin Nutr 1993;58:152-161.

39 Alexy U, Kersting M, Sichert-Hellert W, et al: Macronutrient intake of 3- to 36-month-old German infants and children: results of the DONALD Study. Dortmund Nutritional and Anthropometric Longitudinally Designed Study. Ann Nutr Metab 1999;43:14-22.

40 Daenzer M, Ortmann S, Klaus S, Metges CC: Prenatal high protein exposure decreases energy expenditure and increases adiposity in young rats. J Nutr 2002;132:142-144.

41 Kim S, Mauron J, Gleason R, Wurtman R: Selection of carbohydrate to protein ratio and correlations with weight gain and body fat in rats allowed three dietary choices. Int J Vitam Nutr Res 1991;61:166-179.

42 Jones A, Simson E, Friedman M: Gestational undernutrition and the development of obesity in rats. J Nutr 1984;114:1484-1492.

43 Lucas A, Boyes S, Bloom SR, Aynsley-Green A: Metabolic and endocrine responses to a milk feed in six-day-old term infants: differences between breast and cow's milk formula feeding. Acta Paediatr Scand 1981;70:195-200.

44 Karlberg J, Jalil F, Lam B, et al: Linear growth retardation in relation to the three phases of growth. Eur J Clin Nutr 1994;48(suppl 1):S25-S43. 
45 Hoppe C, Udam TR, Lauritzen L, et al: Animal protein intake, serum insulin-like growth factor I, and growth in healthy 2.5-y-old Danish children. Am J Clin Nutr 2004;80:447-452.

46 Hauner H, Wabitsch M, Zwiauer K, et al: Adipogenic activity in sera from obese children before and after weight reduction. Am J Clin Nutr 1989;50:63-67.

47 Rolland-Cachera MF, Deheeger M, Akrout M, Bellisle F: Influence of macronutrients on adiposity development: a follow-up study of nutrition and growth from 10 months to 8 years of age. Int J Obes Metab Disord 1995;19:573-578.

48 Scaglioni S, Agostoni C, Notaris RD, et al: Early macronutrient intake and overweight at five years of age. Int J Obes Relat Metab Disord 2000;24:777-781.

49 Parizkova J, Rolland-Cachera M: High proteins early in life as a predisposition for later obesity and further health risks. Nutrition 1997;13:818-819.

50 Hoppe C, Molgaard C, Thomsen BL, et al: Protein intake at 9 mo of age is associated with body size but not with body fat in 10-y-old Danish children. Am J Clin Nutr 2004;79:494-501.

\section{Discussion}

Dr. Cong Khanh Nguyen: What are the factors that lead to weight gain during the first week? Are they related to maternal nutrition? The second thing is you addressed the issue of nutrition before 2 years of age as being really critical, not only breastfeeding but also weaning food, the starting time and quality. Somehow it is dealing with protein and energy. So our topic is really interesting with regard to the protein and energy requirements for infancy and childhood. Again it is exactly what we are doing and understand about the requirements of protein. In early childhood protein plays a role in development, but we need to do something with protein in terms of controlling weight gain. It is really important for Viet Nam at the moment; we have to do nutritional controls and also control weight because obesity occurs even in the malnourished population.

Dr. Koletzko: Thank you, I agree this is very important for Viet Nam but also for many other countries around the world that are affected by this double burden of disease, where on the one hand you have a significant proportion of the population that is born with low birth weight under less optimal conditions and then exposed to a diet that predisposes to a high risk of obesity. As we have learned from a number of studies, it appears that if you are born with a low birth weight then at a later age you have a higher risk not only for obesity but also for the metabolic consequences of obesity. There are studies showing that at the same body mass index (BMI) populations born with a low birth weight have a higher body fat content and a much worse metabolic pattern, and a higher disease risk. This is particularly relevant for countries such as Viet Nam where you have this double burden of disease. Now the question whether the first week is relevant; I have my doubts. I have discussed my interpretation of the data published by Stettler et al., but perhaps Dr. Ziegler can also contribute something to that. I have my doubts whether the first week is really so relevant. If you think about the weight change in the first week, there are a lot of variables including water balance, and weight change after birth often is not really the substance of the body. If you look at the first 2 years I think we still have a lot of questions because the analyses we have now, not only from these two studies but also from many other studies, show that there is a relationship between early weight gain and later overweight. But we are really not sure what the critical factors involved are. I think it is a bit premature to come to direct conclusions about intervention. We need to have more data to draw these conclusions. But I agree with you absolutely that it may be a bit short sighted just to look at milk intake, and perhaps there are other factors involved such as complementary feeding. Also non-nutritional factors may be involved which could be relevant. We have seen in our own studies that smoking during early pregnancy also is 
a marked risk factor for children later becoming obese, as opposed to smoking before or after pregnancy. Smoking during the first weeks and months of pregnancy is a strong independent risk factor which points out that not only are dietary and nutritional factors relevant but other factors as well.

Dr. Ziegler: I would like to make two comments regarding the Stettler study. As Dr. Koletzko pointed out, the subjects were studied as infants in Iowa. Although they had a totally different objective, we collaborated with a group in Philadelphia in a telephone follow-up study when the subjects were young adults. We asked the participants about their current weight and height. So their weights are self-reported, with all the reservations one has to have about that. The data showed that overweight status in adulthood was associated with weight gain during the first week of life and with weight gain from birth to 112 days of age, but not from 8 to 112 days of age. We have to conclude that it is really the weight change during the first week of life that predicted later obesity. Now that brings me to my second comment. There is an association between the weight change during the first week of life and later obesity. It does not imply or prove causality, and if there is no causality established, it does also imply that preventive measures aimed at altering weight change during the first week of life should not be expected to be effective, although an effect cannot be ruled out. In other words, if you were to prevent a child from gaining weight in the first week of life, it does not follow that you protect this child from being obese later in life, and i.e. because an association does not in itself establish causality. The studies that you mentioned established an association between breastfeeding in infancy and obesity in childhood, but they did not establish causality.

Dr. Koletzko: I couldn't agree more with you. There is an association with the medium size or modest size effect, and we don't really know where the causality lies and whether that gives an opportunity for preventive intervention. However, if you think about a 20 or $25 \%$ impact on later overweight and obesity on a population basis, this could be very relevant. Therefore I think it is worthwhile to look at this and investigate the potential for improving infant feeding to utilize that preventive effect. But I agree this is a research question, it is not a question that we can turn into policy at present. With respect to the comment on Stettler's study; yes, I agree birth weight is an important factor and it has been shown in many studies that birth weight is a contributing factor to later overweight risk. But I disagree with the conclusion that weight gain during the first week is a most important factor because it just results from using the same dimension, a 100 -gram weight gain from day 0 to 8 and day 0 to 112 . I think this is inappropriate because $100 \mathrm{~g}$ is a huge difference in 1 week but it is a small difference in 112 days. If you use the same proportion, i.e. percentage change in weight as I showed, then it is not the first week of life that is the most predictive time period but the total weight gain in 112 days is more relevant.

Dr. Dewey: We are all fascinated by these recent data on which age interval during the first 2 years might be most critical with regard to weight gain. I think it is important to point out that breastfed infants on the average don't have a net weight gain in the first week of life, they tend to lose a little bit and then come back up, whereas formula-fed infants tend to gain from the very beginning. So it is very intriguing to wonder whether those first 3 days or so of caloric intake, which is minimal in the breastfed infant, might be important metabolically. I think the jury is still out, and I agree with you that we need much more data. I don't know if you have the first week of life in your data set, but the question I have for the whole first 2 years is whether you looked separately at the effect of weight gain during any of those intervals for the breastfed infants in your study as compared to the formula-fed infants? I think there are questions as to whether rapid weight gain in a breastfed infant has the same consequences as it does in a formula-fed infant. 
Dr. Koletzko: That is a very good question. We have included in the publication data obtained on infant feeding at school age (5-6 years). While the data are very reliable on whether the child was breastfed or not breastfed, the question on how long the child was fed exclusively breast milk is not very precise in a retrospective interview at age 6 . Even less precise is the question on when complementary feeding was introduced and the kind of complementary feeding. So we felt a bit uncomfortable about putting too much trust in these details of that study. But now we have the opportunity in the European Childhood Obesity Project to obtain very detailed characteristics of these populations, also in terms of the dietary habits with 3-day records every month in the first year. It will be a much more reliable analysis, and we look forward to seeing what comes from that.

Dr. Haschke: The Nestlé Nutrition Institute recently appointed a committee under the chairmanship of Prof. Lucas to look at all published data on the association between breastfeeding and obesity. The committee pointed out that unfortunately in most meta-analyses there is a selection bias towards positive studies showing an association. However, one study which is continuously neglected is the Iowa Growth Study where more than 400 infants were followed prospectively until 8 years of age; they were either breastfed or formula-fed and all the data on the duration of breastfeeding and type of formula feeding are available. So I would like to ask you to comment on this; first what is the outcome at 8 years of age, and why is this study continuously neglected in meta-analyses?

Dr. Ziegler: The study we conducted was published in 1984 [1]. We followed up a total of 471 children, of whom about one third had been breastfed and two thirds had been formula-fed during the first 4 months of life. All infants participated in the studies in Iowa. Follow-up measurements of height, weight and blood lipids were performed within 2 months of the subjects' 8th birthday. Weight, height and BMI were nearly identical in the formula-fed and breastfed infants; there was no difference whatsoever. The reason why this follow-up study is consistently ignored in all the meta-analyses I think has to do with the fact that our breastfed babies were permitted to receive modest amounts of other foods from an early age, so they were not all exclusively breastfed. But it shows absolutely no difference in adiposity at 8 years. I have to emphasize that we live and work in a small community which is dominated by the university, so most families are somehow affiliated with the university and that may explain why the families are more health conscious than the average family, and it may also explain why on follow-up in the Stettler study the rate of obesity and overweight was unusually low, it was much lower than in the general population. Under these circumstances, when all subjects are health conscious, one sees no association between breast-feeding and late obesity in childhood.

Dr. Koletzko: Let me try to give some comfort as to whether you include 400 infants more or less in a meta-analysis that includes 70,000 or 200,000 infants would not change the result at all. I cannot speak for the meta-analyses published by Harder and by Owen but I can speak for our own meta-analysis and we, in keeping with the concept of evidence-based medicine, defined upfront our criteria for inclusion which included a clear description of exposure, a clear description of results, overweightobesity as odds ratios so that they could be compared, and outcome measured at the age of at least 5 years because we felt that overweight-obesity before the time of obesity rebound doesn't really give a strong predictive result. A journal plot analysis did not give any indication of publication bias. So based on those criteria I think we came to a very clear unbiased conclusion. But I agree, this is the observation of an association, it does not allow any conclusions on cause and effect.

Dr. Butte: Two of the biggest predictors of later obesity in children are maternal and paternal BMI as well as the birth weight of the infant. If you look around the world we tend to put all this early programming into the same pot, and in some populations we 
have very poor gestational weight gain and in other populations, like in United States and Europe, we see very high gestational weight gains, and I think it is unfair to put these in the same basket. What might be occurring to infants born above $4,000 \mathrm{~g}$ is very different from infants born at less than 2,500 g. Could you comment on the effects of both maternal and paternal BMI and gestational weight gain in your studies in Germany?

Dr. Koletzko: As you have probably seen we have done several studies on this issue. In the first study published in the British Medical Journal in 1999, we didn't have data on maternal BMI [2]. In the consecutive studies we collected such data, and what we found is that if we adjust for maternal BMI, e.g. in the Czech study [3], then the effect size is smaller for breastfeeding, but it is still significant in the Czech study with a $20 \%$ risk reduction after adjusting for a variety of factors including maternal BMI. Weight gain in pregnancy is obviously a very relevant candidate to look at, but in our studies we did not have such data included because it is very difficult to get precise measures on that in a retrospective analysis. We recently had a discussion with some obstetricians on the question of whether one come up with recommendations on desirable weight gain, and I started to appreciate how complex this issue is and how difficult it is to come to conclusions. But I would agree with you yes, that is an important area to look at.

Dr. Gomes-Pedro: You have talked about breastfeeding decreasing overweight and obesity at a later age. Can you comment about blood pressure?

Dr. Koletzko: There are some studies in the literature reporting that breastfed populations on average have a slightly lower blood pressure, the difference is in the order of $3 \mathrm{~mm} \mathrm{Hg}$. You may wonder whether $3 \mathrm{~mm} \mathrm{Hg}$ is relevant or not; you may say $3 \mathrm{~mm} \mathrm{Hg}$ is something we can't even measure, why should that be relevant? But on a population basis it seems to be relevant. If you look at the data from the Framingham study then a 3 -mm $\mathrm{Hg}$ difference in average blood pressure really has a relevant effect also on the percentage with critically high blood pressure values, and may have a marked impact on the risk of stroke in the population. The relevant question now is whether this is something that we can influence, and there are two insightful studies that suggest we might. One is a study from the Netherlands [4] in which infants were given different sodium intakes in early life, I believe it was 0.9 and $2.6 \mathrm{mmol}$ sodium, total sodium intake per day for the first 6 months. As might be expected there was a slight difference in blood pressure at the end of the 6 months; but more importantly when these children were revisited 15 years later, there was still a difference in blood pressure of $3 \mathrm{~mm} \mathrm{Hg}$. So that would suggest that protection against very high sodium or sodium chloride intake in the first months of life might be useful and beneficial. The second interesting study was published by Forsyth and coworkers [5]. They revisited children who as infants had participated in randomized trials with and without long-chain polyunsaturated fatty acids in infant formula. When they looked at these children at age 6 years, those children who had received formula with the long-chain polyunsaturated fatty acids again had a 3-mm Hg lower blood pressure; in other words they were closer to breastfed children. However this was a small trial, and I think it is necessary to revisit that question in a somewhat larger, more homogeneous study concept.

Dr. Bozo: My question is about high protein intake during infancy in infant formula and the hypersecretion in insulin; is it related with all kinds of amino acids or is it related with specific amino acids present in the infant formula?

Dr. Koletzko: So far we don't know. If you look at the literature there is a lot of data showing that in animals and in humans, there are certain amino acids which have a particular effect on insulin liberation. When it comes to infants first of all I would say we don't really know whether protein intake affects the described difference in insulin secretion between infants. We have the hypothesis but I don't think we have conclusive data. We have to get them from the European Childhood Obesity Project obviously, but I don't think we can make conclusions at the moment. If that is the case obviously the next question is, is it total protein or is it the quality of protein, and maybe another 
opportunity if the hypothesis proves true is not only to manipulate total protein intake but also to manipulate protein composition to try to adapt the metabolic profile. So I am excited about the question you are asking but I can't offer a perfect answer.

Dr. Rigo: Most of the time in the studies they look at the weight gain. Can you speculate about the quality of the weight gain or length gain during the first part of life and the obesity rate?

Dr. Koletzko: In our study of 4,000 children we saw that length gain between 0 and 6, 6 and 12,12 and 24 months, the totality had a predictive value for later overweight, but the predictive value was less than the value of weight gain. Mind you what we chose as the outcome measure was BMI at 5-6 years. You can ask now is BMI really the best indicator of health risk, and looking back it might have been better to have other measures included to describe obesity worldwide than just BMI, perhaps central obesity, waist-hip ratio or body fat content or whatever might have been more useful than just BMI. In our prospective studies now we are looking at a variety of descriptors of body size and body composition to study some of these questions because BMI in children may not be the best predictor of later health. We need to look at that more closely.

Dr. Kah-Tzay Low: From the epidemiological study by Barker et al. [6] on the relation of birth weight and later death from acute myocardial infarction during adulthood, it was found that those who are born small $(<3.4 \mathrm{~kg})$ and do not reach $10 \mathrm{~kg}$ by 1 year of age are at a higher risk of death from acute myocardial infarction. What are your comments on their conclusion recommending that those who are born with a low birth weight should try to catch up by the first year of life?

Dr. Koletzko: That is a very important question. It points out that it is not only fetal development and fetal growth that are relevant but also postnatal development. It is in contrast with the conclusion of a paper from the same group just published in the New England Journal of Medicine [7] where they propose that it would only be growth after the age of 2 years that would be predictive of later health. The difficulty of course is that some of these are studies performed retrospectively in subjects born in England between 1911 and 1930. So what has induced a high weight gain during the first year in these children is not necessarily what induces weight gain today. I would suspect that, e.g., socioeconomic status would be a very important factor influencing the choice of feeding. I suppose those who were not fed breast milk were fed cow's milk or preparations made from cow's milk rather than what we consider today infant formula. So I would be very careful in drawing conclusions from these observations to what we should do with infants born today.

\section{References}

1 Fomon SJ, Rogers RR, Ziegler EE, et al: Indices of fatness and serum cholesterol at age eight years in relation to feeding and growth during early infancy. Pediatr Res 1984;18:1233-1238.

2 von Kries R, Koletzko B, Sauerwald T, et al: Breast feeding and obesity: cross sectional study. BMJ 199917;319:147-150.

3 Toschke AM, Vignerova J, Lhotska L, et al: Overweight and obesity in 6- to 14-year-old Czech children in 1991: protective effect of breast-feeding. J Pediatr 2002;141:764-769.

4 Geleijnse JM, Hofman A, Witteman JC, et al: Long-term effects of neonatal sodium restriction on blood pressure. Hypertension 1997;29:913-917.

5 Forsyth JS, Willatts P, Agostoni C, et al: Long chain polyunsaturated fatty acid supplementation in infant formula and blood pressure in later childhood: follow up of a randomised controlled trial. BMJ 2003;326:953.

6 Barker DJ, Winter PD, Osmond C, et al: Weight in infancy and death from ischaemic heart disease. Lancet 1989;ii:577-580.

7 Barker DJ, Osmond C, Forsen TJ, et al: Trajectories of growth among children who have coronary events as adults. N Engl J Med 2005;353:1802-1809. 